Pacific Journal of Mathematics

GENERIC COVERING PROPERTIES FOR SPACES OF 


\title{
GENERIC COVERING PROPERTIES FOR SPACES OF ANALYTIC FUNCTIONS
}

\author{
David A. Stegenga and Kenneth Stephenson
}

By a classical result of Fatou, a bounded analytic function on the unit disc $D$, i.e. in the space $H^{\infty}(D)$, has a radial limit at almost every point on $\partial D$. We examine the question of whether this limiting or boundary value lies in the interior or on the boundary of the image domain. We show that the first case is "typical" in the sense that every function in a certain dense $G_{\delta}$-set of $H^{\infty}$ has this property at a.e. boundary point. Several other spaces including the disc algebra and the Dirichlet space are also studied.

1. Introduction. Let $D$ be the unit disc in $\mathbf{C}$. In this paper we consider certain types of covering properties for analytic functions belonging to the disc algebra $A(D)$, the space of bounded analytic functions $H^{\infty}(D)$, and the Dirichlet space $\mathscr{D}$. We prove these properties are generic in the categorical sense; i.e., they hold for functions forming a residual set. A generic property is said to hold for "nearly every" function in the space.

For $f$ analytic in $d$, let $E_{f}$ denote those points $\zeta \in T=\partial D$ where $f(\zeta)$ fails to exist (as a nontangential limit) or where $f(\zeta)$ exists but $f(\zeta) \notin f(D)$. Our main results are summarized in this

THEOREM. (a) The Lebesgue measure $\left|E_{f}\right|$ is zero for nearly every function $f \in H^{\infty}(D)$.

(b) The logarithmic capacity $\operatorname{Cap}\left(E_{f}\right)$ is zero for nearly every function $f \in \mathscr{D}$.

(c) Let $h$ be any Hausdorff measure function. The Hausdorff measure $\Lambda_{h}\left(E_{f}\right)$ is zero for nearly every function $f \in A(D)$.

The theorem says that nearly every function in these spaces maps the boundary of the unit disc into the image of the interior; we might say it buries its boundary values. The size of the exceptional set $E_{f}$ depends upon the space, but our results would seem to be the strongest possible in this regard. One would not expect smaller exceptional sets for $H^{\infty}(D)$ and $\mathscr{D}$, since nontangential limits may not even exist for sets of measure zero and capacity zero, respectively. This doesn't happen in $A(D)$, since these functions are continuous on $\bar{D}$; however, we have shown that by almost any measure of smallness, nearly every function in $A(D)$ has a "small" 
exceptional set. It should be pointed out that a much stronger covering property than that of our theorem may hold in spaces containing unbounded functions. In a sequel to this paper [15], we show that for numerous spaces, including for example the Hardy spaces, nearly every function maps $D$ onto $\mathbf{C}$.

We prove the parts of our theorem in Sections Three, Four, and Five. Our result for $A(D)$ answers a question of Carl Cowen [3] (see also Problem 5.65 of [4]) which arose in his study of Toeplitz operators. He asked for a function $f \in A(D)$ with $\left|E_{f}\right|=0$. An explicit example is described in the remarks at the end of Section Three. Our theorem shows that, in the categorical sense, functions in $A(D)$ without this property are pathological! In $H^{\infty}(D)$, the situation is somewhat reversed: Examples with $\left|E_{f}\right|=0$ are easily found, but the proof that nearly every function has this property is more difficult. It requires a result relating the geometry of plane regions to harmonic measures on their boundaries which may be of independent interest. This is discussed in the Remark at the end of Section Five.

In Section Six we pose some questions regarding other spaces of bounded analytic functions and other types of covering properties. We also discuss some similarities our techniques of proof seem to have with those used with gap power series.

The main ingredient in our proofs is something we call the depth function, $\delta_{f}$, for an analytic function $f$. It measures the size of ramified discs lying in the image surface of $f$ and tells, in an appropriate local sense, how deeply various values are buried in the range of $f$. The depth function is related to more common measures of schlicht discs in the image surface, which have long been of interest in classical analysis, see also [14]. Its properties are developed in the next section after we establish some notation.

This research took place while the second author held a visiting position at the University of Hawaii, Manoa, and he wishes to thank them for their fine hospitality. Both authors thank George Csordas for helpful conversations on the material presented here.

2. The depth function. For $w \in \mathbf{C}$ and $r>0, D(w, r)=\{z: \mid z-$ $w \mid<r\}$. The unit disc is $D=D(0,1)$ with $\partial D=T$, the unit circle. For a Lebesgue measurable set $E \subseteq T,|E|$ denotes its normalized Lebesgue measure.

If $G$ is a plane domain, $H(G)$ denotes the space of analytic functions on $G$. For $f \in H(G)$, let $f(G)=\{w=f(z): z \in G\}$. If $G^{\prime} \subset G$ has compact closure in $G$ we write $G^{\prime} \Subset G$. If $\Gamma \in \mathbf{C}$ is a Borel set, then 
$\omega(z, \Gamma ; G)$ denotes the harmonic measure of $\Gamma \cap \partial G$ with respect to $G$. (see Tsuji [16] Chapter 3.17).

Suppose $f \in H(G), a \in G, b=f(a)$, and $r>0$. We write $\Omega_{a}(r)=$ $\Omega_{a, f}(r)$ for the (connected) component of $f^{-1}(D(b, r))$ in $G$ which contains $a$. If $\Omega_{a}(r) \Subset G$, the argument principle implies that $f$ is a $k$-to-1 map of $\Omega_{a}(r)$ onto $D(b, r)$ for some $k \in \mathbf{Z}^{+}$, the positive integers.

Suppose $f$ is analytic on $D$ and has a nontangential limit $w \in \mathbf{C}$ at some point $\xi \in T$. We write $f(\xi)=w$. Recall, for example that if $f \in$ $H^{\infty}(D)$, then $f(\xi)$ exists for almost all $\xi \in T$.

Definition. Let $G$ be a plane domain. If $f \in H(G)$ and $w \in \mathbf{C}$, the depth function for $f$ on $G$ at $w$ is

$$
\delta(w, f, G)=\sup \left\{t: \Omega_{a}(t) \Subset G, \text { some } a \text { with } f(a)=w\right\} .
$$

When there is no ambiguity about the domain $G$, we write simply $\delta_{f}(w)$.

In geometric terms, $\delta_{f}(w)$ is the supremum of the radii of (ramified) discs centered at $w$ which lie in the image surface of $f$. Thus it tells how deeply $w$ is buried in the range of $f$, but in a local sense, i.e., how the sheets of the image surface cover $w$, rather than a global sense. In particular, the largest disc about $w$ which lies in $f(G)$ will generally have a radius greater than $\delta_{f}(w)$.

In the remainder of this section we establish the main properties of the depth function.

LEMMA 2.1. Let $G$ be a plane domain and $f$ a nonconstant function in $H(G)$.

(a) Either $\delta_{f} \equiv \infty$, or $\delta_{f}: \mathbf{C} \rightarrow[0, \infty)$. In the latter case, $\delta_{f}$ is a Lipschitz function with Lipschitz constant 1.

(b) $\delta_{f}(w)>0$ if and only if $w \in f(G)$

(c) Given $K \subset f(G)$ compact, there exists $\eta>0$ and an open set $G^{\prime} \Subset G$ with $\delta\left(w, f, G^{\prime}\right)>\eta$, for all $w \in K$.

(d) If $g \in H(G)$, then

$$
\sup \left|\delta_{f}(w)-\delta_{g}(w)\right| \leq \sup _{G}|f-g|
$$

with the understanding that if the right side is finite, then $\delta_{f} \equiv \infty$ if and only if $\boldsymbol{\delta}_{g} \equiv \infty$.

Proof. Properties (a) and (b) follow easily from the open mapping theorem. We point out that $\delta_{f} \equiv \infty$ may occur for any domain $G$, even $G=D$. 
To prove (c), let $\tau=\inf _{w \in K} \delta(w, f, G)$. By (a) and (b), $\tau>0$. Choose $\eta$ with $0<\eta<\tau / 4$. There is a finite set $w_{1}, w_{2}, \ldots, w_{n} \in K$ with

$$
K \subset \bigcup_{j=1}^{n} D\left(w_{j}, \eta\right) .
$$

For each $y$, there is a $a_{j} \in G$, with $f\left(a_{j}\right)=w_{j}$ and $\Omega_{a_{j}}(\tau / 2) \Subset G$. Letting $G^{\prime}=\bigcup_{j=1}^{n} \Omega_{a_{1}}(\tau / 2)$, we see $G^{\prime} \Subset G$. Now, suppose $w \in K$ is arbitrary. Then $w \in D\left(w_{j}, \eta\right)$ for some $j$. Because $f$ maps $\Omega_{a_{j}}(\tau / 2)$ onto $D\left(w_{j}, \tau / 2\right)$, there is $a \in \Omega_{a_{j}}(\tau / 2)$ with $f(a)=w$. Since $D(w, \eta) \Subset D\left(w_{j}, \tau / 2\right), \Omega_{a}(\eta)$ $\Subset \Omega_{a_{J}}(\tau / 2) \subset G^{\prime}$. In particular, $\Omega_{a}(\eta) \Subset G^{\prime}$, implying $\delta\left(w, f, G^{\prime}\right)>\eta$.

To prove (d), suppose $\sigma=\sup _{z \in G}|f(z)-g(z)|<\infty$. Assume first that $\delta_{f} \not \equiv \infty$ and $\delta_{g} \not \equiv \infty$ and argue by contradiction. Let $w_{0} \notin \mathbf{C}$ satisfy

$$
\left|\delta_{f}\left(w_{0}\right)-\delta_{g}\left(w_{0}\right)\right|>\sigma .
$$

We may assume that $\delta_{f}\left(w_{0}\right)>\delta_{g}\left(w_{0}\right)$, so (2.1) implies $\delta_{f}\left(w_{0}\right)>\sigma$. Choose $\varepsilon>0$ sufficiently small that $\eta=\delta_{f}\left(w_{0}\right)-\varepsilon \sigma$. For some $a \in f^{-1}\left(w_{0}\right)$, $\Omega=\Omega_{a, f}(\eta) \Subset G$. Since $f$ maps $\partial \Omega$ onto $\partial D\left(w_{0}, \eta\right)$ and $|f-g|<\sigma$ on $\partial \Omega$, we have $g(\partial \Omega) \cap D\left(w_{0}, \eta-\sigma\right)=\varnothing$ and, by Rouche's theorem, $D\left(w_{0}, \eta-\sigma\right) \subset g(\Omega)$. These two facts imply that some component of $g^{-1}\left(D\left(w_{0}, \eta-\sigma\right)\right)$ lies in $\Omega$, hence $\delta_{g}\left(w_{0}\right)>\eta-\sigma$. Now, $\mid \delta_{f}\left(w_{0}\right)-$ $\delta_{g}\left(w_{0}\right)|=| \eta+\varepsilon-\delta_{g}\left(w_{0}\right) \mid<\varepsilon+\sigma$. Since this holds for all small $\varepsilon>0$, we contradict (2.2). A similar argument shows that $\delta_{f} \equiv \infty$ if and only if $\delta_{g} \equiv \infty$.

The domains $G$ which we consider will be $D$ and subsets of $D$. We will use the following results concerning the depth functions for bounded analytic functions on $D$ :

LEMMA 2.2. Let $f, g \in H^{\infty}(D)$ and $\xi \in T$, with $f(\xi)=w_{1}$ and $g(\xi)=$ $w_{2}$. Then

$$
\left|\delta_{f}\left(w_{1}\right)-\delta_{g}\left(w_{2}\right)\right| \leq 2\|f-g\|_{\infty} .
$$

Proof. By Lemma 2.1(a) and (d) we have

$$
\begin{aligned}
\left|\delta_{f}\left(w_{1}\right)-\delta_{g}\left(w_{2}\right)\right| & \leq\left|\delta_{f}\left(w_{1}\right)-\delta_{g}\left(w_{1}\right)\right|+\left|\delta_{g}\left(w_{1}\right)-\delta_{g}\left(w_{2}\right)\right| \\
& \leq\|f-g\|_{\infty}+\left|w_{1}-w_{2}\right| \leq 2\|f-g\|_{\infty} .
\end{aligned}
$$

For $f \in H(D), E_{f}=\{\xi \in T: f(\xi)$ does not exist or $f(\xi) \notin f(D)\}=$ $\left\{\xi \in T: f(\xi)\right.$ does not exist or $\left.\delta_{f}(f(\xi))=0\right\}$. An immediate consequence of Lemma 2 and Fatou's lemma is

LeMma 2.3. Let $f, f_{n} \in H^{\infty}(D)$, with $\left\|f_{n}-f\right\|_{\infty} \rightarrow 0$ as $n \rightarrow \infty$. Then

$$
\limsup _{n \rightarrow \infty}\left|E_{f_{n}}\right| \leq\left|E_{f}\right| \text {. }
$$


3. Disc algebra. The disc algebra $A(D)$ is the space of bounded analytic functions on $D$ which may be extended continuously to $\bar{D}$. It is a Banach space under the supremum norm. Before stating our results we recall the definitions concerning Hausdorff measure (see Chapter 2 [2]). A measure function $h$ is a monotone increasing function defined on $r \geq 0$, with $h(0)=0$. If $E$ is a bounded plane set, we consider coverings of $E$ by a sequence of discs $D_{m}$ with radii $r_{m}$. We define

$$
M_{h}(E)=\inf \sum_{m} h\left(r_{m}\right),
$$

where the infimum is over all such coverings. If we restrict $r_{m} \leq t$ the corresponding lower bound is denoted $\Lambda^{(t)}$, and the limit

$$
\Lambda_{h}(E)=\lim _{t \rightarrow 0} \Lambda^{(t)}
$$

is the Hausdorff measure of $E$. The quantities $M_{h}(E)$ and $\Lambda_{h}(E)$ are zero simultaneously, and it will be more convenient for us to work with $M_{h}$.

THEOREM 3.1. Let $h$ be a measure function. The collection of functions $f$ in $A(D)$ for which $\Lambda_{h}\left(E_{f}\right)=0$ is a dense $G_{\delta}$-set.

With particular choices of $h$, one can show, for example, that those collections for which $\left|E_{f}\right|=0, \operatorname{Cap}\left(E_{f}\right)=0$, the Hausdorff dimension of $E_{f}$ is zero are each dense $G_{\delta}$-sets. Before the proof, we establish a preliminary lemma. Here $\chi$ denotes the identity function $\chi(z)=z, z \in D$.

LEMMA 3.2. Let $\zeta>0$ and $n \geq 2$ be given. Then there exists a function $g=g_{n, \zeta}$ in $A(D)$ with the following properties:

(a) $g(D)=D$,

(b) $g$ is at most 2-to-1,

(c) $\|\chi-g\|_{\infty}<4 \pi / n$

(d) $\{\zeta \in T:|g(\zeta)|=1\}=\bigcup_{j=0}^{n-1} \bar{I}_{j}$ where each $I_{j}$ is an open arc of $T$ with $\left|I_{j}\right|<\zeta$.

Proof of Lemma. We obtain $g$ as a Riemann mapping onto an appropriate simply connected surface $S$ lying over $D$, projected down to $D$. First we construct $S$ :

Let $\Gamma_{0}, \Gamma_{1}, \ldots, \Gamma_{n-1}$ be the arcs in $D$ determined in the following manner. These arcs are congruent, with $\Gamma_{\text {, consisting of a radial segment }}$ of length $1 / n$ ending at the $n$th root of unity $e^{2 \pi l j / n}$ and an attached arc of the circle $\{|z|=1-1 / n\}$. The "doorways" between these circular arcs are each of "angular measure" $2 \pi \zeta$. (We assume that $\zeta<n / 2 \pi$.) 
Let $S_{0}$ denote a copy of $D$ slit along these $\operatorname{arcs} \Gamma_{0}, \Gamma_{1}, \ldots, \Gamma_{n-1}$. We will obtain $S$ by attaching additional surfaces along these slits. For each $t$, $0<t \leq \pi \zeta$, and for each $j=0,1,2, \ldots, n-1$, define

$$
U_{t, j}=\bigcup_{z \in \Gamma_{j}} D(z, t(1-|z|)) \text {. }
$$

We obtain a simply connected surface $S_{t}$ by slitting each $U_{t, j}$ along $\Gamma_{j}$ and attaching it to the corresponding slit in $S_{0}$ with a cross connection. This produces a simple branch point at the interior end of each arc $\Gamma_{j}$. Note that the parameterized family $\left\{S_{t}: t \in[0, \pi \zeta]\right\}$ is a nested collection of surfaces; that is, if $0 \leq t_{1} \leq t_{2} \leq \pi \zeta$, then we will identify $S_{t_{1}}$ with a subset of $S_{t_{2}}$. The surface $S$ which we want is $S_{\tau}$ for an appropriate choice of $\tau, 0<\tau \leq \pi \zeta$.

The unit circle, $T$, is a part of the boundary of each $S_{t}$. Define the harmonic function $\omega_{t}(\cdot)=\omega\left(\cdot, T, S_{t}\right)$ on $S_{t}$. We show that $\omega_{t}(0) \rightarrow \omega_{0}(0)$ as $t \downarrow 0$.

For each $t, 0<t \leq \pi \zeta$, let $u_{t}$ be the harmonic function on $S_{0}$ whose boundary values are these:

$$
u_{t}(\zeta)= \begin{cases}1 & \text { if } \xi \in T \\ \max \left\{\omega_{t}\left(S_{1}\right), \omega_{t}\left(S_{2}\right)\right\} & \text { if } \xi \in \bigcup_{j=0}^{n-1} \Gamma_{j}\end{cases}
$$

where the point $S_{1}$ and $S_{2}$ are the two points of $S_{t}$ lying over $\xi$.

For all $t \in(0, p i \zeta]$ we have

$$
\omega_{0} \leq\left.\omega_{t}\right|_{S_{0}} \leq u_{t} \quad \text { on } S_{0} .
$$

For each point $\xi \in \bigcup_{j=0}^{n-1} T$, which is not an endpoint of one of these arcs, $u_{t}(\xi)$ clearly goes to 0 as $t \searrow 0$. Thus, $u_{t}(0) \searrow \omega_{0}(0)$, and the inequality above implies $\omega_{t}(0) \searrow \omega_{0}(0)$ as $t \searrow 0$.

If $J$ is the union of the $n$ open arcs (the "doorways") of the circle $\{|z|=1-1 / n\}$ which lie in $S_{0}$, then

$$
\omega_{0}(0)=\omega\left(0, T, S_{0}\right)<\omega\left(0, J, D\left(0,1-\frac{1}{n}\right)\right) .
$$

This last value is exactly $2 \pi n \zeta / 2 \pi=n \zeta$. We therefore choose $\tau, 0<\tau \leq$ $\pi \zeta$, so that

$$
\omega_{\tau}(0)<n \zeta .
$$

Our surface $S$ is taken to be $S_{\tau}$.

Let $p: S \rightarrow D$ be the natural projection, let $\phi$ be a one-to-one conformal map of $D$ onto $S$, and define $g=p \cdot \phi$. We may choose $\phi$ so 
that $g(0)=0$. Properties (a) and (b) are clear from the construction of $S$. The function $g$ is in $A(D)$ because each boundary point of $S$ has a neighborhood which is conformally equivalent under $p$ to a Jordan region in the plane.

Properties (c) and (d) will follow from the $n$-fold rotational symmetry of $S$. Define

$$
J_{j}=\left\{e^{i \theta}: \frac{2 \pi j}{n} \leq \theta \leq \frac{2 \pi(j+1)}{n}\right\} .
$$

The boundary of $S$ breaks into the $n$ congruent, connected pieces

$$
B_{j}=J_{j} \cup\left(\partial U_{\tau, j}\right), \quad j=0,1, \ldots, n-1 .
$$

By a standard symmetry argument, $g$ satisfies

$$
g(\lambda z)=\lambda g(z)
$$

for $\lambda=e^{2 \pi i / n}$. Therefore, $T=\bigcup_{j=0}^{n-1} g^{-1}\left(B_{j}\right)$, and each set $g^{-1}\left(B_{j}\right)$ is an arc of angular measure $2 \pi / n$. Composing with a rotation if necessary, we may assume that $J_{j}=g^{-1}\left(B_{j}\right)$, for $j=0,1, \ldots, n-1$. Since $J_{j} \subset B_{j}$, and the diameter of $B_{j}$ is at most $4 \pi / n$ (c) follows.

Finally, since conformal maps preserve harmonic measure,

$$
\left|\left\{e^{i^{\theta}}:\left|g\left(e^{i^{\theta}}\right)\right|=1\right\}\right|=\omega\left(0, t, S_{\tau}\right)=\omega_{\tau}(0)<n \zeta .
$$

But $\left\{e^{i^{\theta}}:\left|g\left(e^{i^{\theta}}\right)\right|=1\right\}$ clearly consists of $n$ congruent closed arcs, $\bar{I}_{0}, \ldots, \bar{I}_{n-1}$, hence $\left|I_{j}\right|<\zeta, j=0, \ldots, n-1$.

Proof of Theorem 3.1. Define the set

$$
\mathscr{G}_{N}=\left\{f \in A(D): M_{h}\left(E_{f}\right)<1 / N\right\},
$$

for $N=1,2, \ldots$. Clearly $f \in \bigcap_{N} \mathscr{G}_{N}$ if and only if $M_{h}\left(E_{f}\right)=0$, hence if and only if $\Lambda_{h}\left(E_{f}\right)=0$. It therefore suffices by the Baire category theorem to prove that each $\mathscr{G}_{N}$ is open and dense.

To prove it is open, fix $f \in \mathscr{G}_{N}$. There is a countable collection of discs $D_{m}$ with radii $r_{m}$ so that $E_{f} \subset \cup_{m} D_{m}$ and $\Sigma_{m} h\left(r_{m}\right)<1 / N$. Since $\xi \rightarrow \delta_{f}(f(\xi))$ is continuous on $T=\partial D$.

$$
\eta=\inf \left\{\delta_{f}(f(\xi)): \xi \in T \backslash \bigcup_{m} D_{m}\right\}
$$

is positive. If $g \in A(D)$ with $\|f-g\|_{\infty}<n / 2$, then Lemma 2 implies $\delta_{g}(g(\xi))>0$ for $\xi \in T \backslash \bigcup_{m} D_{m}$; that is, $E_{g} \subset \cup_{m} D_{m}$. Hence $g \in \mathscr{G}_{N}$, and $\mathscr{G}_{N}$ is open. 
To prove density, fix $f \in A(D)$ and $\varepsilon>0$. Since $f$ is uniformly continuous on $\bar{D}$, there exists $\tau>0$ so that $\left|f\left(z_{1}\right)-f\left(z_{2}\right)\right|<\varepsilon$ if $z_{1}$, $z_{2} \in \bar{D}$ with $\left|z_{1}-z_{2}\right|<\tau$. Choose $n \geq 2$ with $4 \pi / n<\tau$ and choose $\zeta>0$ with $n \cdot h(\pi \zeta)<1 / N$. Let $g$ be the corresponding function $g_{\eta, \zeta}$ from Lemma 4. Clearly $f \circ g \in A(D)$. Also, by Lemma 3.2(c), $\|\chi-g\|_{\infty}<$ $4 \pi / n<\tau$. Therefore, $\|f-f \circ g\|_{\infty}=\|f \circ \chi-f \circ g\|_{\infty}<\varepsilon$. It remains only to prove that $f \circ g \in \mathscr{G}_{N}$.

If $\xi \in T$ and $|g(\xi)|<1$, then by Lemma 3.2(a), $g(\xi) \in g(D)$ and hence $(f \circ g)(\xi) \in(f \circ g)(D)$. Consequently, $E_{f \circ g} \subseteq\{\xi \in T:|g(\xi)|=1\}$. By Lemma 3.2(d), this latter set is contained in $\bigcup_{j=0}^{n-1} \bar{I}_{j}$ with $\left|I_{j}\right|<\zeta$. The diameter of each $I$ is less than $2 \pi \zeta$ (because $|\cdot|$ denotes normalizes Lebesgue measure), so we can find discs $D_{j}, j=0,1, \ldots, n-1$, of radius $\pi \zeta$ so that $E_{f \circ g} \subset \cup_{j} D_{j}$. By our choice of $\zeta, n \circ h(\pi \zeta)<1 / N$ so $M_{h}\left(E_{f \circ g}\right)$ $<1 / N$ and $f \circ g \in \mathscr{G}_{N}$.

REMARKS. (a) In some sense this is the best possible result concerning the metric properties of $E_{f}$ for $f \in A(D)$. For if $\Lambda_{h}\left(E_{f}\right)=0$ for every measure function $h$, then $E_{f}$ would be countable, a clear impossibility. By a result of Besicovitch [1], assuming the continuum hypothesis there are uncountable measure sets this "small," but they cannot be Borel; our $E_{f}$ is closed.

(b) A concrete example of a function $f \in A(D)$ with $\left|E_{f}\right|=0$ can be obtained by extending the construction technique used in the proof of Lemma 4. However, here is a more straightforward construction.

EXAMPLE. Our function $f$ will be of the form $f \equiv g^{3}$, where $g$ is a conformal mapping of $D$ onto a simply connected domain $\Omega \subset D$. The domain $\Omega$ is obtained from $D$ by removing countably many radial slits ending at $T$. Specifically, at each primitive $2^{n}$-root of unity $\xi$ remove the slit ending at $\xi$ of length $2^{-n}, n=1,2,3, \ldots$

The boundary of $\Omega$ is locally connected, so the conformal map $g$ : $D \rightarrow \Omega$ is in $A(D)$. [Theorem 9.8, 11] It follows that $f \in A(D)$. Also, $f(D)=D$ since $\Omega=g(D)$ contains at least one of the cube roots of $z$ for every $z \in D$. To show $\left|E_{f}\right|=0$, it therefore suffices to show that $|E|=0$, where $E=\{\xi \in T:|g(\xi)|=1\}$. It is clear that no point of $E$ is a twist point for $g$. By McMillan's twist point theorem [7], $g$ is conformal at almost all points of $E$. This means that if $|E|>0$, then there is some point $\xi \in E$ so that $\Omega=g(D)$ contains Stolz regions of arbitrarily large angular opening at $g(\xi) \in T$. However, our construction guarantees that no Stolz region of opening greater than $\operatorname{Tan}^{-1}(\pi)$ lies in $\Omega$. Therefore, $|E|=0$. 
4. Dirichlet space. The Dirichlet space $\mathscr{D}$ is the collection of functions $f \in H(D)$ with

$$
\|f\|_{\mathscr{D}}=\left(\iint_{D}\left|f^{\prime}(z)\right|^{2} d m_{2}(z)\right)^{1 / 2} \rightarrow|f(0)|<\infty,
$$

where $m_{2}$ denotes normalized area measure on $D$. This defines a norm under which $\mathscr{D}$ is a Banach space. The elements $f \in \mathscr{D}$ have nontangential limits, which we continue to denote $f(\zeta)$, at all points $\xi \in T$ with the possible exception of a subset of capacity zero. Also, we have

$$
\operatorname{Cap}\{\xi \in T:|f(\xi)|>t\} \leq \frac{\|f\|_{\mathscr{D}}^{2}}{t^{2}}, \quad t>0 .
$$

(See [13] for the preliminaries for this section.)

THEOREM 4.1. The collection of functions $f$ in $\mathscr{D}$ for which $\operatorname{Cap}\left(E_{f}\right)=0$ is a dense $G_{\delta}$-set.

We need a preliminary lemma. For $g \in H(D)$ and $w \in \mathbf{C}, n(g, w)$ denotes the cardinality, counting multiplicities, of $g^{-1}\{w\}$.

LeMma 4.2. Let $g_{k}: D \rightarrow D$ be analytic, $k \in \mathbf{Z}^{+}$, with $\left\|\chi-g_{k}\right\|_{\infty} \rightarrow 0$ as $k \rightarrow \infty$ and

$$
\sup _{|w|<1} n\left(g_{k}, w\right) \subseteq M \in \infty, \text { for all } k .
$$

Then $f \circ g_{k} \rightarrow f$ in $\mathscr{D}$ as $k \rightarrow \infty$ for each $f \in \mathscr{D}$.

Proof of Lemma. For $0<r<1$,

$$
\begin{aligned}
\iint_{|z|<1} \mid f & -\left.\left(f \circ g_{k}\right)^{\prime}\right|^{2} d m_{2} \\
\leq & \iint_{|z|<r}\left|f^{\prime}(z)-f^{\prime}\left(g_{k}(z)\right) g_{k}^{\prime}(z)\right|^{2} d m_{2}(z) \\
& +\iint_{r \leq|z|<1}\left|f^{\prime}(z)\right|^{2} d m_{2}(z)+\iint_{r \leq|z|<1}\left|f^{\prime}\left(g_{k}(z)\right) g_{k}^{\prime}(z)\right|^{2} d m_{2}(z) \\
& \leq A_{k, r}+B_{r}+C_{k, r}
\end{aligned}
$$

For $r$ close to $1, B_{r}$ is small since $\iint_{D}\left|f^{\prime}\right|^{2} d m_{2}<\infty$. Since $g_{k} \rightrightarrows \chi$ on $D$, $\lim _{k \rightarrow \infty} A_{k, r}=0$. Finally,

$$
C_{k, r}=\iint_{g_{k}(\{r \leq|z|<1\})}\left|f^{\prime}(w)\right|^{2} n\left(g_{k}, w\right) d m_{2}(w) .
$$


Write $m=\min _{r \leq|z|<1}\left|g_{k}(z)\right|$, then

$$
C_{k, r} \leq M \iint_{m \leq|w|<1}\left|f^{\prime}(w)\right|^{2} d m_{2}(w)
$$

Since $m \rightarrow r$ as $k \rightarrow \infty, \overline{\lim }_{k \rightarrow \infty} C_{k, r} \leq M \circ B_{r}$. This completes the proof.

Proof of Theorem 4.1. Define the set

$$
\mathscr{G}_{N}=\left\{f \in \mathscr{D}: \operatorname{Cap}\left(E_{f}\right)<\frac{1}{N}\right\}
$$

for $N=1,2,3, \ldots$ It is enough to show that each $\mathscr{G}_{N}$ is open and dense.

Open. Fix $f \in \mathscr{G}_{N}$. We claim that there exists a compact subset $K \subset \partial D$ for which the restriction of $f$ to $K$ is a continuous function and $\operatorname{Cap}(T \backslash K)<1 / 2 N$. This is a fairly standard type result which can be proved (in considerable generality) using Theorem 4(iii) [8] along with the integral representation of functions in $\mathscr{D}$ [Lemma 2.1, 13]. Since $f$ is continuous on $K$ so is $\delta_{f} \circ f$ and hence $E_{f} \cap K=\left\{\zeta \in K: \delta_{f} \circ f(\zeta)=0\right\}$. The outer regularity of capacities then gives a $\delta>0$ for which $K_{\delta}=\{\zeta \in$ $\left.K: \delta_{f} \circ f(\zeta) \geq \delta\right\}$ also satisfies $\operatorname{Cap}\left(T \backslash K_{\delta}\right)<1 / 2 N$. By Lemma 2.1(c) there is a subdisc $D^{\prime} \Subset D$ and $\eta>0$ for which $\delta\left(f(\zeta), f, D^{\prime}\right)>\eta$ for all $\zeta \in K_{\delta}$. By taking $\|f-g\|_{\mathscr{D}}$ sufficiently small we have $|f(z)-g(z)|<$ $\eta / 2$ for $z \in D^{\prime}$ and hence by Lemma 2.1(d) $\delta\left(f(\zeta), g, D^{\prime}\right)>\eta / 2$ for all $\zeta \in K_{\delta}$. Observe that if $\zeta \in K_{\delta}$ and $|f(\zeta)-g(\zeta)|<\eta / 2$ then $\delta\left(g(\zeta), g, D^{\prime}\right)>0$ so that $\zeta \notin E_{g}$. But by (4.1) we may assume that $|f(\zeta)-g(\zeta)|<\eta / 2$ for all $\zeta \in \delta D$ except for a set with capacity less that $1 / 2 N$ (take $\|f-g\|_{\infty}$ smaller if necessary). By the subadditivity property of capacities the capacity of $E_{\delta}$ is less than $\operatorname{Cap}\left(T \backslash K_{\delta}\right)$ plus $1 / 2 N$ which is less than $1 / N$ and hence $\mathscr{G}_{N}$ is open.

Density. Let $f \in \mathscr{D}$ and $\varepsilon>0$. By Lemma 3.2 and Lemma 4.2 we can choose $g$ so that $\left\|f \circ g-f_{\infty}\right\|<\varepsilon$ and $\operatorname{Cap}\left(E_{g}\right)<1 / N$. Since $E_{f \circ g} \subset E_{g}$ it follows that $f \circ g \in \mathscr{G}_{N}$ and hence $\mathscr{G}_{N}$ is dense. Thus, the proof of Theorem 4.1 is complete.

5. The space $\boldsymbol{H}^{\infty}$. The main result in this section is

THEOREM 5.1. The collection of functions $f \in H^{\infty}$ for which $\left|E_{f}\right|=0$ is a dense $G_{\delta}$-set.

The proof is more difficult than those of the previous theorems, since the composition techniques used there no longer work. A prime ingredient 
is a preliminary result relating the geometry of plane domains to the harmonic measures of their boundaries. This result should be of independent interest (see the Remark at the end of the section). First some needed notation.

Let $\Omega \subset \mathbf{C}$ be open. For $w \in \mathbf{C}, r>0$, let $\theta^{*}(w, r, \Omega)$ denote the angular measure of the largest arc of

$$
\{z:|z-w|=r\} \cap \Omega,
$$

with $\theta^{*}(w, r, \Omega)=\infty$ if $\{z:|z-w|=r\} \subset \Omega$. We will have occasion to consider $w=\infty$; circles about $\infty$ correspond to circles centered at 0 , so we let:

$$
\theta^{*}(\infty, r, \Omega)=\theta^{*}\left(0, \frac{1}{r}, \Omega\right)
$$

for $r>0$.

Define $\Theta=\Theta_{\Omega}: \mathbf{C} \times(0, \infty) \rightarrow[0, \infty]$ by $\Theta(w, r)=\theta^{*}(w, r, \Omega)$. It is easily verified that $\Theta$ is lower semicontinuous. For $\alpha>0, \Lambda_{\alpha}$ denotes Hausdorff measure using the measure function $h(r)=r^{\alpha}, r \geq 0$.

LeMmA 5.2. Let $\Omega \subset \mathrm{C}$ and $\alpha>\frac{1}{2}$ be given. If $\Gamma \subset \partial \Omega$ is a Borel set with $\Lambda_{\alpha}(\Gamma)<\infty$, then all points $w \in \Gamma$ satisfy

$$
\varlimsup_{r \rightarrow 0}\left\{\theta^{*}(r, w, \Omega)\right\} \geq \frac{\pi}{\alpha}
$$

with the possible exception of a set having harmonic measure zero with respect to $\Omega$.

Proof. We assume $0 \in \Omega$. Our first step is to show that when $w_{0} \in \partial \Omega$ and (5.1) fails, then

$$
\lim _{r \rightarrow 0} \frac{\omega\left(0, D\left(w_{0}, r\right), \Omega\right)}{r^{\alpha}}=0 .
$$

We begin by letting $w_{0}=\infty$, in which case $D\left(w_{0}, r\right)$ denotes the set $\{|w|>1 / r\}$. For $r>0$ let $\Omega_{r}=\{w \in \Omega:|w|<1 / r\}$. By the maximum principle,

$$
\omega\left(0, D(\infty, r), \Omega_{r}\right) \geq \omega(0, D(\infty, r), \Omega) .
$$

If (5.1) fails, then there is some $\beta>\alpha$ with

$$
\varlimsup_{r \rightarrow 0}\left\{\theta^{*}(\infty, r, \Omega)\right\}<\frac{\pi}{\beta}<\frac{\pi}{\alpha} .
$$

Choose $r_{0}$ with $\theta^{*}(\infty, r, \Omega)<\pi / \beta$ for $0<r<r_{0}$. That is,

$$
\theta^{*}(0, t, \Omega)<\frac{\pi}{\beta} \quad\left(t>r_{0}^{-1}=t_{0}\right) .
$$


By an estimate of Tsuji [Theorem III.67, 16]

$$
\omega\left(0, D(\infty, r), \Omega_{r}\right) \leq C \exp \left\{-\pi \int_{t_{0}}^{1 / 2 r} \frac{d t}{t \theta^{*}(0, t, \Omega)}\right\}
$$

for $1 / r>2 t_{0}$, some constant $C$. By (5.4),

$$
\frac{\pi}{\theta^{*}(0, t, \Omega)}>\beta \quad\left(t>t_{0}\right)
$$

is

$$
\omega\left(0, D(\infty, r), \Omega_{r}\right) \leq C \exp \left\{-\beta \int_{t_{0}}^{1 / 2 r} \frac{d}{t}\right\} \leq C^{\prime} r^{\beta}
$$

Since $\beta>\alpha$, this and (5.3) imply (5.2) for $w_{0}=\infty$. We may now prove (5.2) for the general case that $w_{0} \in \partial \Omega, w_{0} \in \mathbf{C}$. If $-\bar{w}_{0}^{-1} \in \Omega$, we simply transform to problem with the linear fractional transformation

$$
\varphi(z)=\frac{z+\bar{w}_{0}^{-1}}{z-w_{0}}, \quad z \in \mathbf{C}^{\infty} .
$$

This maps $-\bar{w}_{0}^{-1}$ to 0 and $w_{0}$ to $\infty$. Circles centered at $w_{0}$ are transformed to circles centered at $\infty$ (i.e., circles centered at 0 ); moreover, the angular measures of corresponding arcs are preserved. Also, harmonic measure is preserved. We therefore obtain

$$
\lim _{r \rightarrow 0} \frac{\omega\left(-\bar{w}_{0}^{-1}, D\left(w_{0}, r\right), \Omega\right)}{r^{\alpha}}=0 .
$$

By Harnach's inequality,

$$
\lim _{r \rightarrow 0} \frac{\omega\left(0, D\left(w_{0}, r\right), \Omega\right)}{r^{\alpha}}=0 .
$$

If $-\bar{w}_{0}^{-1} \in \Omega$, then for $r_{0}$ sufficiently small we may replace $\Omega$ by

$$
\Omega^{\prime}=\Omega \cup\left\{\left|w-w_{0}\right|>r_{0}\right\}
$$

with $-\bar{w}_{0}^{-1} \in \Omega^{\prime}$. We have shown that (5.2) holds for $\Omega^{\prime}$. For $0<r<r_{0}$, the maximum principle implies

$$
\omega\left(0, D\left(w_{0}, r\right), \Omega\right) \leq \omega\left(0, D\left(w_{0}, r\right), \Omega\right),
$$

so (5.2) holds for $\Omega$ also.

We may assume $\Lambda_{\alpha}(\Gamma)=0$. For $\Lambda_{\alpha}(\Gamma)<\infty$ implies $\Lambda_{\alpha+\varepsilon}(\Gamma)=0$ for $\varepsilon>0$. Having proven our lemma's conclusion for $\alpha+\varepsilon$, it will hold for $\alpha$ also. 
Let $\Gamma^{\prime}$ consist of the points $w \in \Gamma$ for which (5.1) fails. Because $w \rightarrow \Theta(w, r, \Omega)$ is lower semicontinuous, a routine argument shows $\Gamma^{\prime}$ is a Borel set. It remains only to show that $\omega\left(0, \Gamma^{\prime}, \Omega\right)=0$.

By (5.2), for each $w \in \Gamma^{\prime}$, we may choose a radius $\zeta(w)$ so that

$$
\frac{\omega(0, D(w, r), \Omega)}{r^{\alpha}}<1, \quad 0<r \leq \zeta(w) .
$$

Define $\Gamma_{n}=$ closure $\left\{w \in \Gamma^{\prime}: \zeta(w)>1 / n\right\} \cap \Gamma^{\prime}, n=2,3,4, \ldots$ Each $\Gamma_{n}$ is a Borel set, and $\Gamma^{\prime}=\cup_{n} \Gamma_{n}$. We show $\omega\left(0, \Gamma_{n}, \Omega\right)=0, n=2,3, \ldots$

Let $\varepsilon>0$ be given. since $\Lambda_{\alpha}\left(\Gamma_{n}\right) \leq \Lambda_{\alpha}(\Gamma) \leq 0$, we can find open discs $D_{\nu}$ with radii $r_{\nu}<1 / 2 n$, so that $\Gamma_{n} \subset \bigcup_{\nu} D_{\nu}$ and $\sum_{\nu}\left(2 r_{\nu}\right)^{\alpha}<\varepsilon$. We may assume, of course, that each $D_{\nu}$ intersects $\Gamma_{n}$. Choose $w_{\nu} \in \Gamma_{n} \cap D_{\nu}$ with $\zeta\left(w_{\nu}\right)>1 / n>2 r_{\nu}$. If $\tilde{D}_{\nu}=D\left(w_{\nu}, 2 r_{\nu}\right)$ then by (5.5),

$$
\omega\left(0, D\left(w_{\nu}, 2 r_{\nu}\right), \Omega\right)<\left(2 r_{\nu}\right)^{\alpha} \text {. }
$$

In particular,

$$
\omega\left(0, \Gamma_{h}, \Omega\right) \leq \sum_{\nu} \omega\left(0, \tilde{D}_{\nu}, \Omega\right)<\sum_{\nu}\left(2 r_{\nu}\right)^{\alpha}<\varepsilon .
$$

Since $\varepsilon>0$ was arbitrary, $\omega\left(0, \Gamma_{h}, \Omega\right)=0$. This concludes the proof of the lemma.

Proof of Theorem 5.1. Define the sets

$$
\mathscr{G}_{N}=\left\{f \in H^{\infty}(D):\left|E_{f}\right|<\frac{1}{N}\right\}
$$

for $N=1,2,3, \ldots$ Clearly $f \in \bigcap_{N} \mathscr{G}_{N}$ if and only if $\left|E_{f}\right|=0$. By the Baire category theorem it suffices to prove that each $\mathscr{G}_{N}$ is open and dense.

That $\mathscr{G}_{N}$ is open follows directly from Lemma 2.3. To show density, it is enough to verify the following

Claim. There is a universal constant $C<1$ so that given any nonconstant $f \in H^{\infty}(D)$ and $\varepsilon>0$, there exists $g \in H^{\infty}(D),\|f-g\|_{\infty}<\varepsilon$, with $\left|E_{g}\right| \leq C\left|E_{f}\right|$.

We show that $C=1-2^{-14}$ works. Let $f \in H^{\infty}(D)$ be given. If $\left|E_{f}\right|=0$, there is nothing to show, so assume $\left|E_{f}\right|>0$. The function $g$ will be of the form

$$
g(z)=f(z)+\sigma \cdot h(z) z^{n}, \quad z \in D
$$

where $\sigma>0, n \in \mathbf{Z}^{+}$, and $h \in H^{\infty} w(D),\|h\|_{\infty} \leq 1$, are appropriately chosen. 
Begin with $\sigma$. By choosing $\sigma<\varepsilon$, we see $\|f-g\|_{\infty} \leq \sigma<\varepsilon$. From Lemma 2.2, we see that $\sigma$ may be made smaller yet, if necessary, to ensure that

$$
\left|E_{g} \backslash E_{f}\right|<2^{-1 / 4}\left|E^{f}\right|
$$

Henceforth, $\sigma$ is fixed.

Next, we construct $h$ : Let $\Omega=f(D)$, and apply Lemma 5.2 with $\Gamma=\partial \Omega$ and $\alpha=2$. Since $\Gamma$ has finite area, $\Lambda_{2}(\Gamma)<\infty$. Thus, at all points $w \in \partial \Omega$, with the exception of a set of harmonic measure zero, there is a sequence $r_{j} \rightarrow 0$ with $\theta^{*}\left(r_{j}, w, \Omega\right)>\pi / 4$. Although these $r_{j}$ depend on $w$, they can be perturbed slightly using the function $\Theta=\Theta_{\Omega}$ defined earlier. Let $r_{1}, r_{2}, \ldots$ be an enumeration of the rationals in $(0, \sigma)$. Inductively define $\Gamma_{1}, \Gamma_{2}, \ldots \subseteq \partial \Omega$ by

$$
\Gamma_{j}=\left\{w \in \partial \Omega \backslash \bigcup_{k=1}^{j-1} \Gamma_{k}: \Theta\left(r_{j}, w\right)>\frac{\pi}{4}\right\} .
$$

Since $\Theta$ is lower semicontinuous, each $\Gamma_{j}$ is a Borel set and, by the result from Lemma 5.2,

$$
\omega\left(z, \partial \Omega \backslash \bigcup_{j} \Gamma_{j} ; \Omega\right) \equiv 0, \quad z \in \Omega .
$$

Define $F_{j}=\left\{\zeta \in T: f(\zeta) \in \Gamma_{j}\right\}, j=1,2, \ldots$ Each $F_{j}$ is a measurable subset of $E_{f}$, and Löwner's Theorem [16, p. 322] and (5.8) imply $\left|E_{f} \backslash \bigcup_{j} F_{j}\right|=0$. Choose $j_{0}$ so large that $\left|\bigcup_{j=1}^{j_{0}} F_{j}\right|>\frac{1}{2}\left|E_{f}\right|$. By a routine argument, there is a compact set $K \subset \Omega$ so that, for each $j=1,2, \ldots, j_{0}$, the set

$$
E_{j}=\left\{\zeta \in F_{j}: \theta^{*}\left(r_{j}, \zeta, \text { Int } K\right)>\frac{\pi}{8}\right\}
$$

satisfies

$$
\left|E_{j}\right|>\frac{1}{2}\left|F_{j}\right| \text {. }
$$

We now choose $h$ to be the outer function [12, Chap. 17] with

$$
|h(\zeta)|= \begin{cases}\frac{r_{j}}{\sigma} & \text { for } \zeta \in E_{j}, j=1,2, \ldots, j_{0}, \\ 1, & \zeta \in T \backslash \bigcup_{j=1}^{j_{0}} E_{j} .\end{cases}
$$

Since each $r_{j}<\sigma, h \in H^{\infty}(D)$ with $\|h\|_{\infty} \leq 1$. Henceforth, $h$ remains unchanged. 
Finally, we must choose the integer $n$. Apply Lemma 1(c) to $f$, taking $K$ to be the compact set in $F(D)$ found above. This gives a radius $R$, $0<R<1$, and $\eta>0$ so that

$$
\delta(w, f, D(0, R))>\eta, \quad \text { for } w \in K .
$$

Take $n$ sufficiently large that $R^{n}<\eta$. By (5.6),

$$
\sup _{|z| \leq R}|f(z)-g(z)|<\eta \text {. }
$$

Along with Lemma 2.1(d) and (5.9), this implies that $g(D)$ contains $K$.

For each $\zeta \in \bigcup_{j=1}^{j_{0}} E_{j}$, with the possible exception of a set of measure zero where $|h|$ does not take its prescribed radial limit, there is an arc $J(\zeta) \subset T$ of angular measure $\pi / 8$ with

$$
[f(\zeta)+\sigma h(\zeta) \lambda] k \in K, \quad \text { for } \lambda \in J(\zeta) .
$$

By the pigeon hole principle, there exists some arc $J \subset T$ of angular measure $\pi / 16$ such that

$$
E^{\prime}=\left\{\zeta \in \bigcup_{j=1}^{j_{0}} E_{j}: J \subset J(\zeta), \text { and }(5.10) \text { holds }\right\}
$$

satisfies

$$
\left|E^{\prime}\right| \geq \frac{1}{32}\left|\bigcup_{j=1}^{j_{0}} E_{j}\right|
$$

By the generalized Riemann-Lebesgue lemma (see Chap. II, Thm. 4.15 [18]).

$$
\frac{1}{2 \pi} \int_{0}^{2 \pi} \chi_{E^{\prime}}\left(e^{i \theta}\right) \chi_{J}\left(e^{i n \theta}\right) d \theta \rightarrow|J|\left|E^{\prime}\right|=\frac{1}{32}\left|E^{\prime}\right|
$$

as $n \rightarrow \infty$. Increase our $n$, if necessary, so that

$$
\frac{1}{2 \pi} \int_{0}^{2 \pi} \chi_{E^{\prime}}\left(e^{i \theta}\right) \chi_{J}\left(e^{i n \theta}\right) d \theta>\frac{1}{64}\left|E^{\prime}\right|
$$

This implies that the set

$$
E^{\prime \prime}=\left\{\zeta \in E^{\prime}: \zeta^{n} \in J\right\}
$$

satisfies

$$
\left|E^{\prime \prime}\right|>\frac{1}{64}\left|E^{\prime}\right|
$$


Now, if $\zeta \in E^{\prime \prime} \subset E^{\prime}$, then $\zeta^{n} \in J$ and $J \subset J(\zeta)$, so (5.10) implies

$$
g(\zeta)=f(\zeta)+\sigma h(\zeta) \zeta^{n} \in K \subset g(D) .
$$

Therefore, $E^{\prime \prime} \cap E_{g}=\varnothing$, while $E^{\prime \prime} \subset E_{f}$. Since

$$
\left|E^{\prime \prime}\right|>\frac{1}{64}\left|E^{\prime}\right|>\left(\frac{1}{64}\right)\left(\frac{1}{32}\right)\left|\bigcup_{j=1}^{j_{0}} E_{j}\right|>\left(\frac{1}{64}\right)\left(\frac{1}{32}\right)\left(\frac{1}{4}\right)\left|E_{f}\right|,
$$

we see that

$$
\left|E_{g} \cap E_{f}\right|<\left(1-2^{-13}\right)\left|E_{f}\right| \text {. }
$$

Along with (5.7) this gives

$$
\left|E_{g}\right|<\left(1-2^{-4}\right)\left|E_{f}\right| \text {. }
$$

This proves the claim with $C=1-2^{-14}$, and completes the proof of the theorem.

REMARKs. (a) The idea behind the proof is this: By Lemma 5.2, most points of $\partial f(D)$ have many directions in which a small displacement will push them into $f(D)$. We modified $f$ by adding a "displacement" function, namely $\sigma h(z) z^{n}$, which frequently added a vector in the right direction to bury some of the boundary. The parameter $\sigma$ kept $f$ and $g$ close in $H^{\infty}(D)$, while $h$ gave our displacements the correct lengths based on the geometry of $\Omega$. The $n$ played two roles: It "randomized" the directions of the displacement vectors, so that a certain fixed proportion of them would hit $\Omega$. But it also "froze" enough $\Omega$ in place so that $\Omega$ would still be there when the displacement arrived! The randomizing effect is very similar to what occurs with gapped power series and leads to some of their covering behavior, see [17], [5], and [9].

(b) Results similar to Lemma 5.2 have appeared in the literature. McMillan [6] considered simply connected domains $\Omega$ and showed that for all $w \in \partial \Omega$, with the possible exception of a set with harmonic measure zero, $\varlimsup_{r \rightarrow 0} \theta^{*}(r, w, \Omega) \geq \pi$. Lemma 5.2 gives the bound $\pi / 2$ for arbitrary domains. The best bound for general domains seems to be an open question. Øksendal [10] also has related results, although he does not concern himself with the "largest arc" as we do. However, he does conjecture that harmonic measure on $\partial \Omega$ is singular with respect to $\Lambda_{\alpha}$ for $\alpha>1$. Were this the case, Lemma 5.2 would imply McMillan's result for arbitrary domains. Note that the method of Lemma 5.2 will also yield area results like those of McMillan and Øksendal. In light of the above we conjecture that $\varlimsup_{r \rightarrow 0} \theta^{*}(\zeta, r) \geq \pi$, for arbitrary domains $G$, where $\zeta \in \partial G$ is chosen outside an exception set of zero harmonic measure. 


\section{REFERENCES}

[1] A. S. Besicovitch, Concentrated and rarified sets of points, Acta Math., 62 (1934), 289-300.

[2] L. Carleson, Selected Problems on Exceptional Sets, Van Nostrand, 1967.

[3] C. C. Cowen, The commutant of an analytic Toeplitz operator, Trans. Amer. Math. Soc., 239 (1978), 1-31.

[4] D. A. Brannan and J. G. Clunie, Editors, Aspects of Contemporary Complex Analysis, Academic Press, London, 1980.

[5] W. H. J. Fuchs, On the zeros of power series with Hadamard gaps, Nagoya Math. J., 29 (1967), 167-174.

[6] J. E. McMillan, On the boundary correspondence under conformal mapping, Duke Math. J., 37 (1970), 725-739.

[7] __ Boundary behavior of a conformal mapping, Acta Math., 123 (1969), 43-67.

[8] N. G. Meyers, $A$ theory of capacities for potentials of functions in Lebesgue classes, Math. Scand., 26 (1970), 255-292.

[9] T. Murai, The value-distribution of lacunary series and a conjecture of Paley, Ann. Inst. Fourier, Grenoble 31, 1 (1981), 135-156.

[10] B. Øksendal, Brownian motion and sets of harmonic measure zero, Pacific J. Math., 95 (1981), 179-192.

[11] Ch. Pommerenke, Univalent Functions, Vendenhoeck \& Ruprecht, Göttingem, 1975.

[12] W. Rudin, Real and Complex Analysis, McGraw-Hill, New York, 1966.

[13] D. A. Stegenga, Multipliers of the Dirichlet space, Illinois J. Math., 24 (1980), 113-139.

[14] d. A. Stegenga and K. Stephenson, A geometric characterization of analytic functions with bounded mean oscillation, J. London. Math. Soc., 24 (1981), 243-254.

[15] _ Generic covering properties for spaces of analytic functions, II, preprint.

[16] M. Tsuji, Potential Theory in Modern Function Theory, Maruzen Co., Tokyo, 1959.

[17] G. Weiss and M. Weiss, On the Picard property of lacunary power series, Studia Math., 22 (1963), 221-245.

[18] A. Zygmund, Trigonometric Series, 2nd Edition, Cambridge University Press, New York, 1959.

Received November 23, 1983 and in revised form February 7, 1984. Both authors are supported by grants from the National Science Foundation.

UNIVERSITY OF HAWAII AT MANOA

HoNOLULU, HI 96822

AND

UNIVERSITY OF TENNESSEE

KNOXVILLE, TN 37916 



\section{PACIFIC JOURNAL OF MATHEMATICS EDITORS}

\author{
V. S. VARADARAJAN (Managing Editor) \\ University of California \\ Los Angeles, CA 90024 \\ Charles R. DePrima \\ California Institute of Technology \\ Pasadena, CA 91125 \\ R. FINN \\ Stanford University \\ Stanford, CA 94305
}

\author{
HeRmanN FlaschKa \\ University of Arizona \\ Tucson, AZ 85721 \\ Ramesh A. Gangolli \\ University of Washington \\ Seattle, WA 98195 \\ ROBION KIRBY \\ University of California \\ Berkeley, CA 94720
}

C. C. MOORE

University of California

Berkeley, CA 94720

H. SAMELSON

Stanford University

Stanford, CA 94305

HAROLD STARK

University of California, San Diego

La Jolla, CA 92093

ASSOCIATE EDITORS
R. ARENS
E. F. BECKENBACH
B. H. NeUmanN
F. WOLF
K. YosHIDA (1906-1982)

\section{SUPPORTING INSTITUTIONS}

UNIVERSITY OF ARIZONA

UNIVERSITY OF BRITISH COLUMBIA

CALIFORNIA INSTITUTE OF TECHNOLOGY

UNIVERSITY OF CALIFORNIA

MONTANA STATE UNIVERSITY

UNIVERSITY OF NEVADA, RENO

NEW MEXICO STATE UNIVERSITY

OREGON STATE UNIVERSITY
UNIVERSITY OF OREGON

UNIVERSITY OF SOUTHERN CALIFORNIA

STANFORD UNIVERSITY

UNIVERSITY OF HAWAII

UNIVERSITY OF TOKYO

UNIVERSITY OF UTAH

WASHINGTON STATE UNIVERSITY

UNIVERSITY OF WASHINGTON 


\section{Pacific Journal of Mathematics}

Vol. 119, No. $1 \quad$ May, 1985

Maurice Chacron, Nonisotropic unitary spaces and modules with

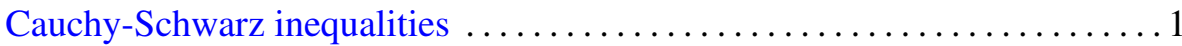

Myriam Dechamps-Gondim, Françoise Piquard and H. Queffélec, On

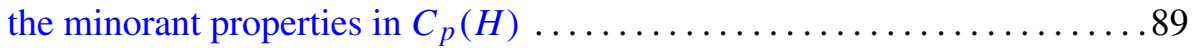

Klaus Floret and V. B. Moscatelli, On bases in strict inductive and projective limits of locally convex spaces .................... 103

Norman Joseph Goldstein, Degenerate secant varieties and a problem on

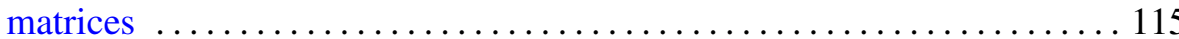

Harold Morris Hastings and Stefan Waner, $G$-bordism with singularities

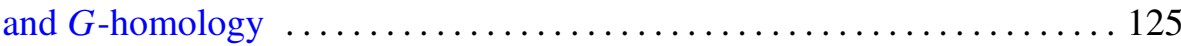

Takesi Isiwata, Clopen realcompactification of a mapping ........... 153

Hisao Kato, Concerning hyperspaces of certain Peano continua and strong

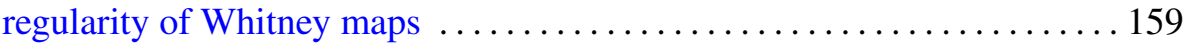

Elyahu Katz and Sidney Allen Morris, Free products of topological groups

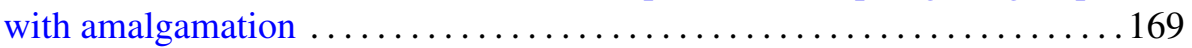

Kyewon Koh Park, Nice dense subsets for ergodic flows and Bernoulli

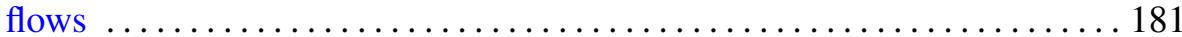

Francis Pastijn and Peter George Trotter, Lattices of completely regular

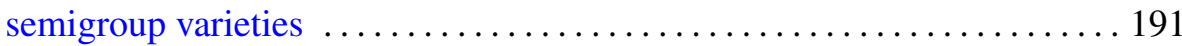

Rae Michael Andrew Shortt, Reticulated sets and the isomorphism of analytic powers

David A. Stegenga and Kenneth R. Stephenson, Generic covering properties for spaces of analytic functions

M. V. Subba Rao and R. Sitaramachandra Rao, On some infinite series of

L. J. Mordell and their analogues 